Neurosurg Focus 19 (1):Intro, 2005

\title{
Endoscopic techniques in skull base surgery
}

\author{
Amin B. Kassam, M.D. \\ Department of Neurosurgery, Minimally Invasive Neurosurgery Center, and Center for Cranial Base \\ Surgery, University of Pittsburgh Medical Center, Pittsburgh, Pennsylvania
}

Since its inception, neurosurgery has benefited from close collaborations with other specialties. Each succeeding decade has been marked with key advances based on these collaborations. The original collaboration with otolaryngology led to the refinement of microsurgical techniques, and the collaboration between neurosurgery and radiation oncology evolved into the current concepts of stereotactic radiosurgery. In the 1990s, the interaction between neurosurgery and interventional radiology created the discipline of endovascular neurosurgery, and the intense collaboration between otolaryngology, plastic surgery, and neurosurgery originated the field of skull base surgery. Each of these advances has been characterized by crosspollination that allowed the integration of parallel technological developments as they occurred within each field.

Physicians using these new treatment algorithms strove to make them the standard of care based on improved clinical outcomes. In each instance, a demonstration of feasibility and an initial learning curve was followed by a period of validation and the popularization of those reproducible elements that resulted in improved outcomes. These events eventually resulted in new treatment paradigms that incorporated the original and the emerging technologies and procedures. Over the past several years, similar collaborations and cross-pollination have spearheaded the concept of minimally invasive surgery along the entire neuraxis, with endoscopy being used as the primary visualization tool ("endoneurosurgery").

We are currently at a critical stage in the evolution of this discipline. Over the past decade, significant advances in technology have been integrated to enhance the feasibility of this evolving field. In this issue of Neurosurgical Focus the contributors will present the techniques of endoneurosurgery in a systematic way, through a series of modular approaches. This work is based on modules that are built on a sound anatomical foundation, which will be outlined using cadaveric studies. We will then present the representative surgical techniques and clinical applications for each of these modular approaches. In this issue we will also focus on key concerns of intraoperative management related to hemostasis and reconstruction. Critical concepts such as team surgery and the use of a stepwise approach to learning will be emphasized. Avoidance of complications is optimized by the surgeon's sequential progression through the modules.

Once its feasibility is established, the eventual role of endoneurosurgery, its indications, and its limitations will be determined through outcome studies. Preliminary outcome data from multiple institutions, although still reflecting the initial learning curve, indicate that these techniques have the potential to improve outcomes in wellselected patients. We have the unique opportunity, as these innovative techniques are advanced globally, to collect outcome data prospectively to provide definite answers. International collaboration accomplished using shared databases will allow for the collection of outcome data with both efficiency and accuracy.

Finally, this issue of the journal will also provide a glimpse into emerging technologies and new techniques with potential clinical applications that are undefined. 